\title{
'Return to the New Normal': Innovative Solutions for Safe Ponseti Casting in Clubfoot Clinics during the COVID-19 Pandemic
}

\author{
Alaric Aroojis ${ }^{1}\left[\right.$ D $\cdot$ Vigneshwaran Pragadeeswaran ${ }^{1}$
}

Received: 10 August 2020 / Accepted: 15 September 2020 / Published online: 10 October 2020

(c) Indian Orthopaedics Association 2020

\begin{abstract}
Clubfoot treatment using the Ponseti method has been severely impacted as a result of lockdown measures implemented by several countries during the COVID-19 pandemic. As clubfoot clinics begin phased re-opening, we describe various innovative techniques to protect patients and healthcare providers during delivery of clubfoot treatment services. In addition to standard social distancing measures and sanitization protocols, we report a simple and innovative technique of 'barrier casting' or 'ring-fencing' to potentially reduce the risk of droplet infection and airborne transmission during clubfoot casting and tenotomy. A transparent, disposable plastic drape is set up as a curtain and serves as an impermeable barrier between the caregiver and healthcare provider performing the casting or tenotomy. The plastic drape is cheap (<US\$1.5), easily available and can be used effectively even in low- and middle-income countries which have a high patient load. We have utilized these innovative techniques in 43 consecutive patients since clinic re-opening and are satisfied that they are effective, economical and easily reproducible even in countries with limited resources. Innovative techniques, which have the potential to reduce the risk of disease transmission, will help in the rapid recovery of clubfoot treatment services as countries emerge from months of lockdown.
\end{abstract}

Keywords COVID-19 $\cdot$ Clubfoot $\cdot$ Ponseti casting $\cdot$ Barrier $\cdot$ Ring-fencing $\cdot$ Innovation

\section{Introduction}

The Coronavirus disease pandemic (COVID-19), caused by the SARS-CoV-2 virus has spread all across the globe, affecting 213 countries since January 2020. In an attempt to arrest community transmission and slow the spread of the virus, several countries initiated complete or partial lockdowns of their populations. India, the 2 nd most populous country in the world [1], announced an immediate and total lockdown of its 1.35 billion population on 24th March 2020, when the number of confirmed positive COVID-19

Alaric Aroojis and Vigneshwaran Pragadeeswaran have equal contributions.

Alaric Aroojis

aaroojis@gmail.com

Vigneshwaran Pragadeeswaran

drvignesh.92@gmail.com

1 Department of Paediatric Orthopaedics, Bai Jerbai Wadia Hospital for Children, Acharya Donde Marg, Parel, Mumbai, Maharashtra 400012, India cases in the country had reached $\sim 500$ [2]. Overnight, all non-essential private and public transport services including road, rail, metro and suburban trains, and domestic and international flights were suspended; educational institutions and commercial establishments were shuttered; and all social, political, cultural and religious gatherings were prohibited [2]. Along with banks, pharmacies, grocery shops \& other essential services, hospitals were permitted to functionbut at reduced capacity and mainly to provide emergency and essential patient care. Routine outpatient services were severely curtailed and elective surgeries and procedures were encouraged to be postponed or cancelled. The lockdown was extended in 3 more phases on 14th April, 3rd May and 17th May 2020, with some relaxations permitted in non-COVID hotspots (designated as 'orange' and 'green' zones) starting early May [3]. From 1st June 2020, the Ministry of Home Affairs (MHA) announced phased re-opening of the nation with gradual lifting of restrictions, except in the so-called 'red containment zones' [4]. A group of researchers from the University of Oxford designated India's lockdown as 'one of the most stringent in the world', scoring a perfect 100 
on the Oxford COVID-19 Government Response Tracker (OxCGRT) [5].

\section{Impact of India's Lockdown on Clubfoot Treatment}

Clubfoot is a common congenital musculoskeletal deformity that can result in significant long-term disability, if left untreated. With an approximate incidence of 1.24 per 1000 live births [6], it is estimated that $\sim 35,000$ children are born with clubfoot in India every year. Since 2002, the Ponseti method has supplanted surgery as the standard of care for clubfoot across most of India. A national clubfoot programme using the Ponseti method has been developed since 2009, as a result of public-private partnerships between State Governments' Ministries of Health and international non-profit organizations. Our institution, which is an urbanbased, tertiary care, children's hospital runs a high-volume Clubfoot Clinic and has treated 1432 children with clubfeet from August 2011-March 2020 (an average of 175 new clubfoot cases per year) as part of this collaborative partnership. The sudden imposition of lockdown caused a severe disruption of clubfoot treatment services at our institution, as patients were unable to travel to the clinic. Since clubfoot treatment is considered an elective procedure, new patients were not registered in the clinic and all casting and bracing visits were suspended. At the time of the lockdown in March 2020, our clinic had registered 11 new patients, 62 patients were in the casting phase and 58 patients were in brace follow-up (total of 131 patients under active treatment).

\section{Resumption of Clubfoot Services Upon Phased Relaxation of Lockdown}

As of 8 th June 2020, outpatient clubfoot treatment services have partially resumed at our clinic. Since our city has the highest number of COVID-19 cases in the state, which in turn has the highest number of cases in the country $(5,15,332$ cases out of a county-wide total of 2.2 million cases as of 10th August 2020) [7], several services including public transport are still suspended. As we gradually open up clinical services in a phased manner, our clinic has devised some innovative measures to permit safe delivery of clubfoot treatment while protecting our patients and doctors from risk of exposure to infection. We wish to share some of the safety measures that we have devised to protect our healthcare workers and patients in a high-volume clubfoot setting.
1. Creating a triage area at the hospital entrance to screen patients and caregivers with mandatory temperature checks. Patients with fever and/or respiratory symptoms are referred to the Fever and Flu Clinic.

2. Calling clubfoot patients by appointment only, so as to ensure that there is no over-crowding in the waiting area and social distancing norms can be maintained. Since ours is a public hospital, the Paediatric Orthopaedic outpatient clinics were frequently over-crowded with up to 40-50 patients per clinic in pre-COVID times ( $>10,000$ outpatient visits per year).

3. Permitting only one caregiver with the baby inside the treatment room during casting, tenotomy and bracing.
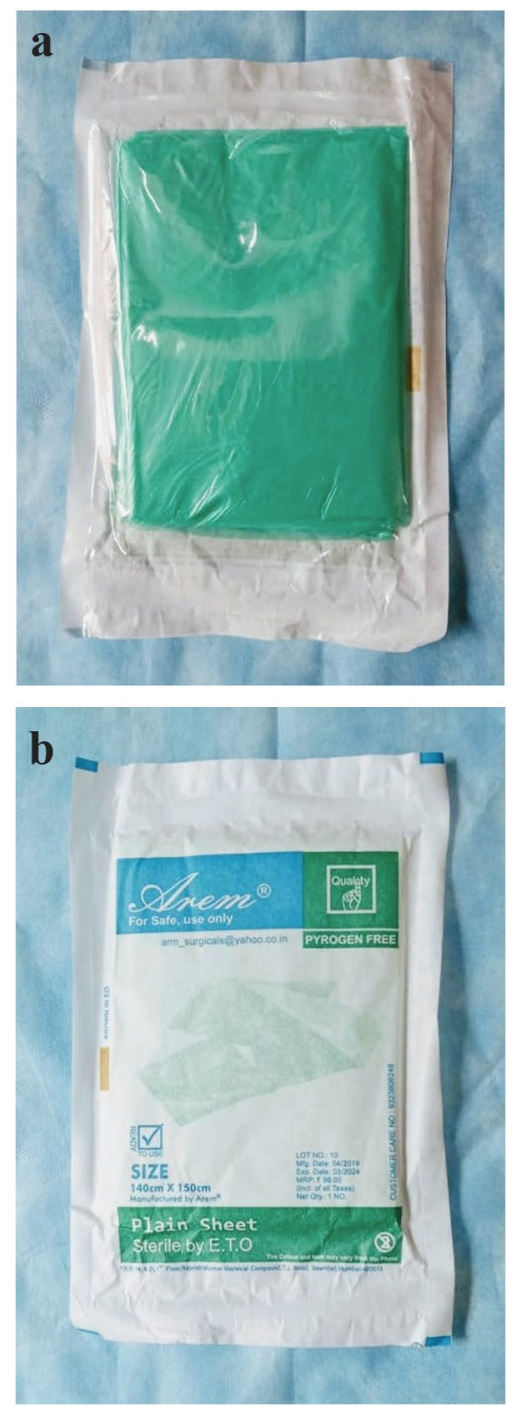

Fig. 1 Photo showing disposable plastic drape used for "barrier casting' 
Fig. 2 Photo showing set-up of 'barrier casting'. a Photo showing two small holes cut out in the plastic drape. b Photo showing positioning of the child with legs inserted through the holes in the drape and a snug fit around the upper thighs
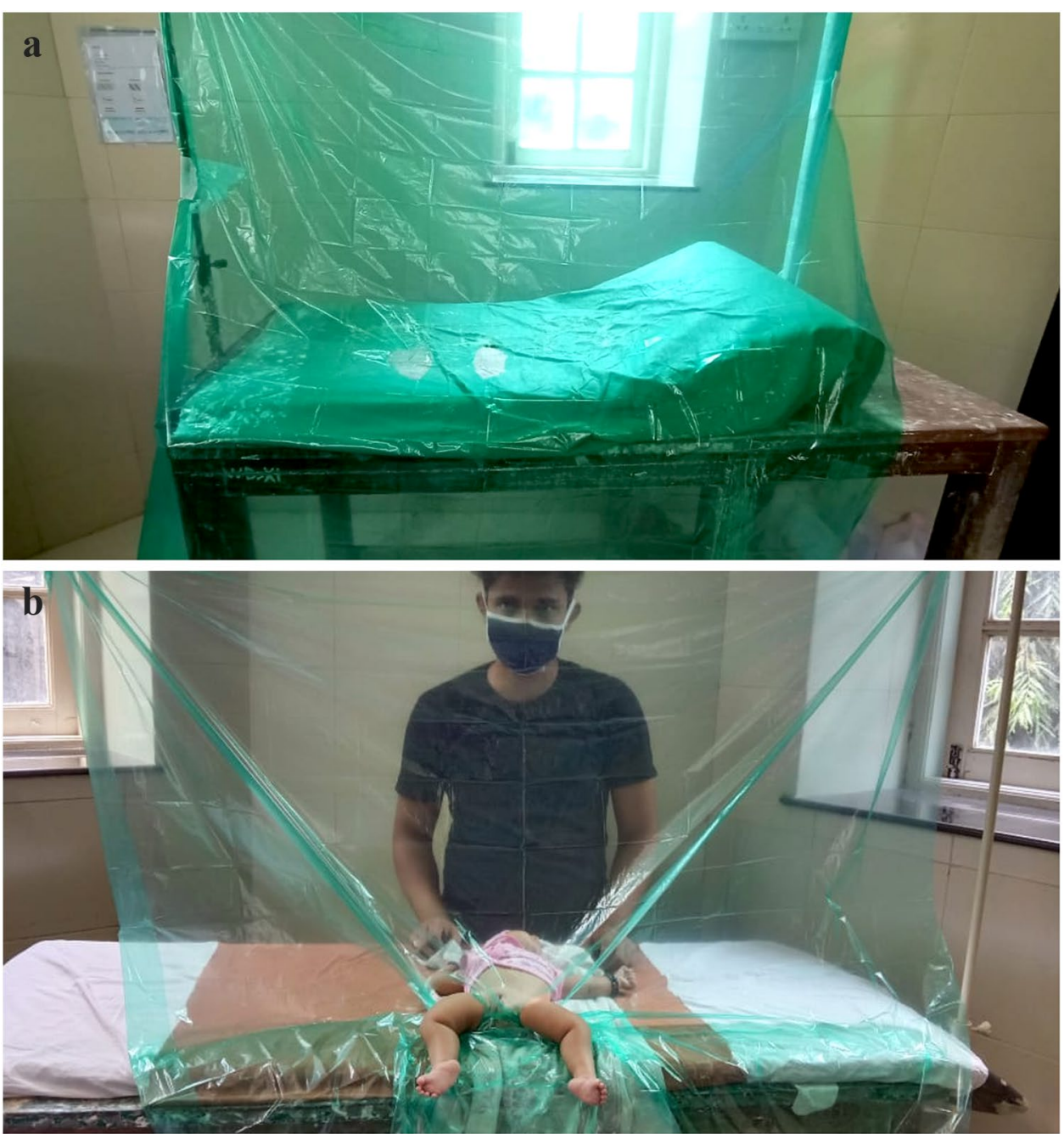

4. Providing required personal protection equipment (PPE) to healthcare providers (doctor/assistant) in the form of FFP2 mask, plastic face shield, cloth gown and nonsterile gloves, since the main modes of transmission are considered to be droplet infection and airborne transmission of the virus [8].

5. Ensuring caregiver wears a face-covering or mask and performs hand sanitization before entering the treatment room.

6. Practicing 'barrier casting' or 'ring-fenced casting'. This is a novel and innovative measure which seeks to isolate the healthcare providers from the patient and caregiver by providing a fluid-impermeable plastic barrier between the two.

\section{Technique}

A $140 \mathrm{~cm} \times 150 \mathrm{~cm}$ impermeable, disposable plastic drape (Arem, Mumbai, India) (Fig. 1a, b) supported by I.V. stands on either side of the casting table is held up as a curtain between the patient and the doctor performing the casting. Two holes, which are roughly the circumference of the child's upper thigh, are carefully cut out in the drape and the child's legs are slid through the holes allowing a snug fit of the plastic drape on the upper thighs (Fig. 2a, b). The proximal part of the drape is held up vertically on supporting stands, thus creating an impermeable but transparent barrier, between the patient and caregiver on one side of the barrier and the healthcare provider and casting assistant on the other side (Fig. 3a, b). The distal part of the drape lies beneath the legs of the patient and falls over the edge of the table so as to protect the caster 
Fig. 3 Photo showing positions of patient, caregiver and providers. a Photo showing position of child and caregiver. b Photo showing position of caregiver and providers on either side of the barrier drape a
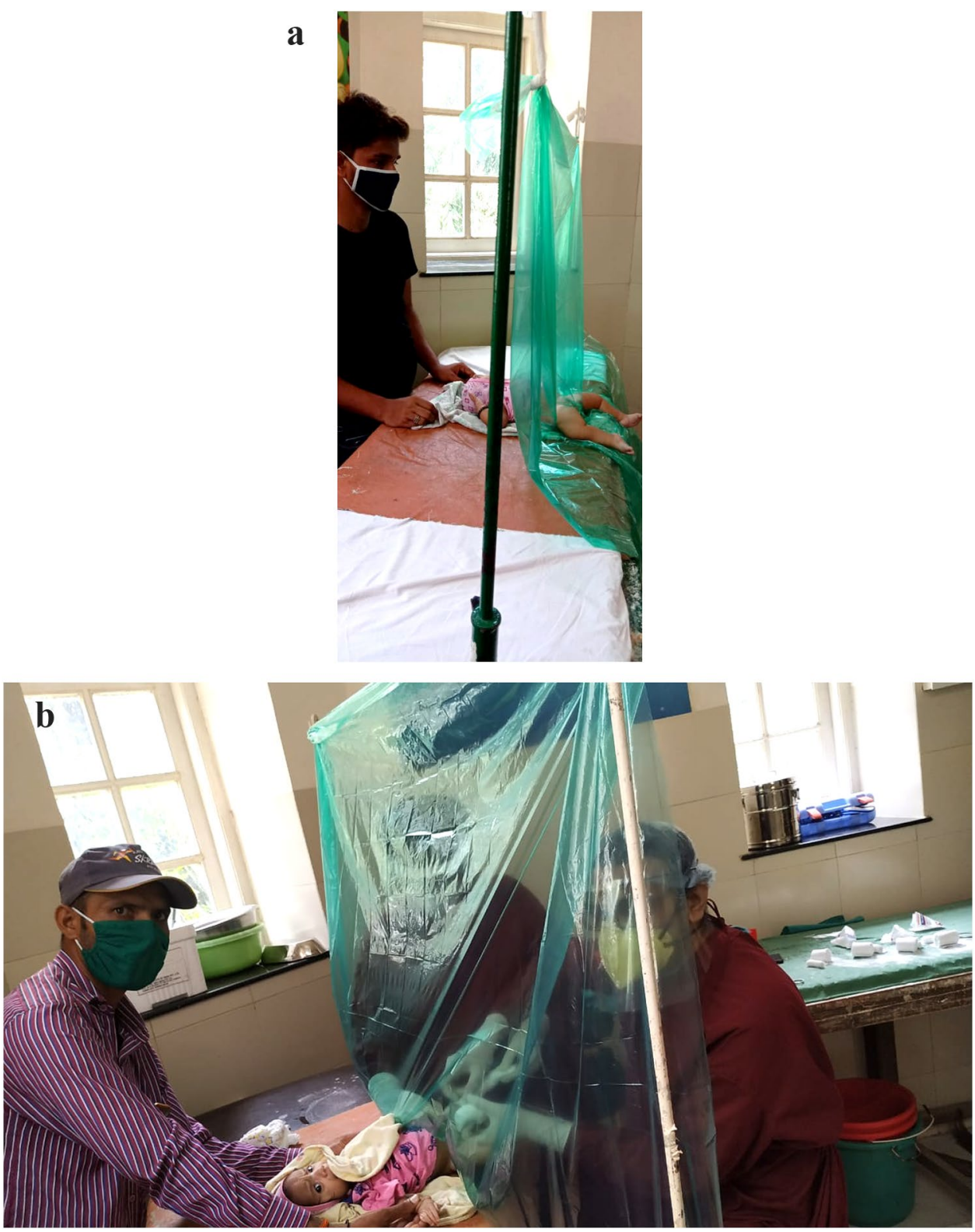

from wet plaster spillage during the casting and moulding process. The transparent plastic drape not only creates a physical barrier between patient and provider, but also permits the provider to keep a watch over the baby to avoid undue force and discomfort during manipulation and casting (Fig. 4a, b). The disposable plastic drape is easily available at a cost of $\sim$ Rupees 100 (US\$1.30) and should be discarded and replaced with a new one between consecutive patients.

\section{Results}

We have utilized these innovative techniques in 43 consecutive patients since clinic re-opening and are satisfied that they are effective, economical and easily reproducible even in countries with limited resources. 
Fig. 4 Photo showing final set-up during manipulation and casting. a Photo from the side showing optimal position of child and caregiver. b Photo showing effective barrier protection during casting
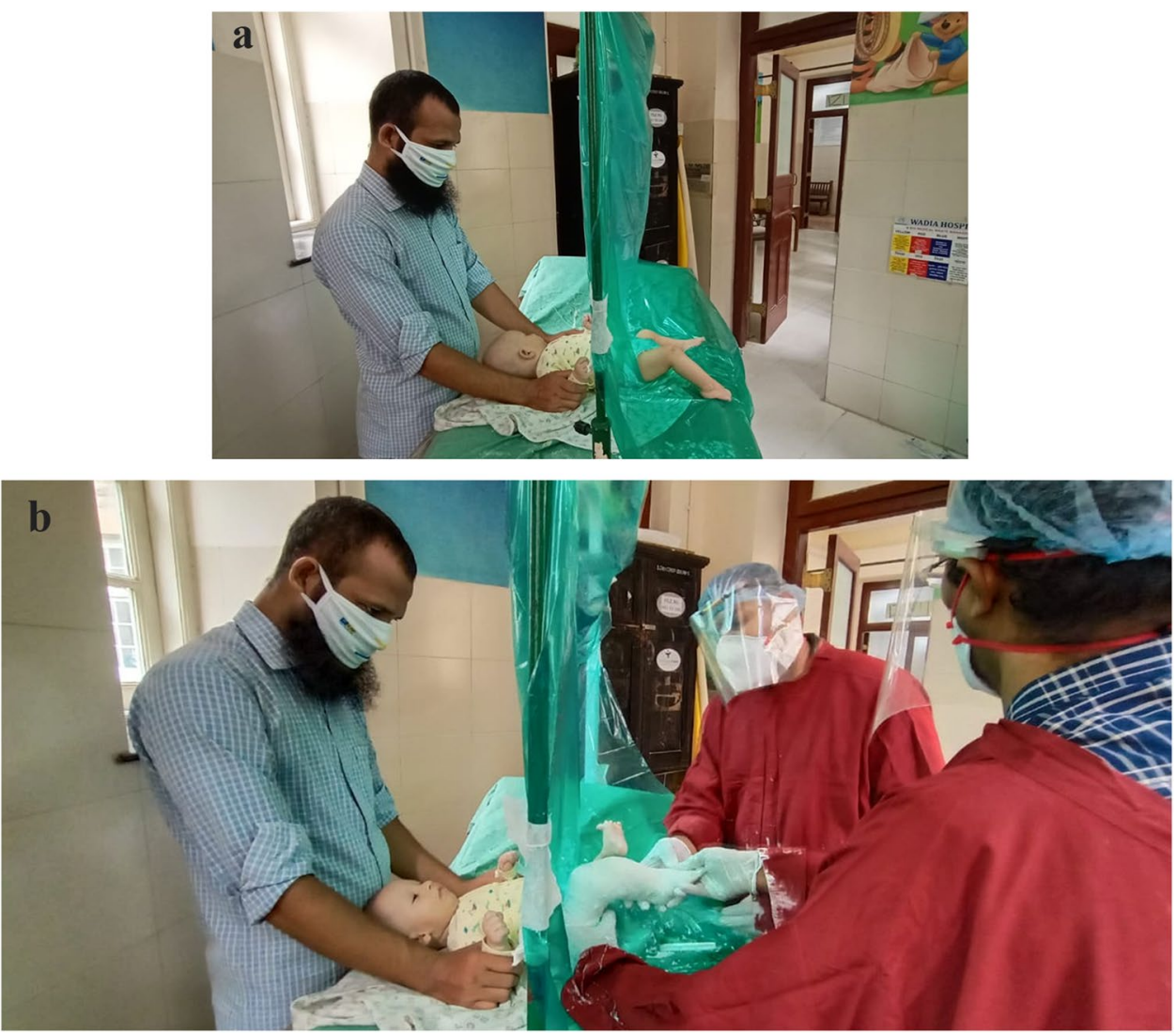

\section{Conclusions}

We are confident that these simple and practical tips, using cheap and easily available materials will go a long way in protecting patients and healthcare workers from the risk of disease transmission, as we begin the slow and arduous task of resuming full clinical operations.

Acknowledgements The authors thank the administration of Bai Jerbai Wadia Hospital for Children, Mumbai, India for permission to utilize hospital data.

Author contributions Alaric Aroojis was responsible for the study conception and design. Material preparation and clinical photos were contributed by Vigneshwaran P. The first draft of the manuscript was written by Alaric Aroojis and both authors read and approved the final manuscript.

Funding This study has no funding support.

\section{Compliance with Ethical Standards}

Conflict of Interest The authors declare that they have no conflict of interest.

Ethical Approval This article does not contain any studies with human or animal subjects performed by the any of the authors.
Informed Consent Informed consent was obtained from all individual participants included in the study.

Consent to Publish The authors affirm that participants provided informed consent for publication of the images in Figs. 2, 3 and 4.

\section{References}

1. World Bank 2018 https://data.worldbank.org/indicator/SP.POP. TOTL?locations=IN. Accessed 10 Aug 2020.

2. Guidelines on the measures to be taken by Ministries/Departments of Government of India, State/Union Territory Governments and State/Union Territory Authorities for containment of COVID-19 Epidemic in the country. Annexure to Ministry of Home Affairs Order No. 40-3/2020-D dated 24.03.2020. https://www.mha.gov. in/notifications/circulars-covid-19.

3. Consolidated Guidelines of MHA on Lockdown measures on containment of COVID-19. https://www.mha.gov.in/media/whats -new? page $=1$.

4. MHA Order Dt. 30.5.2020 with guidelines on extension of lockdown in Containment Zones and phased reopening. No.403/2020-DM-I(A). https://www.mha.gov.in/media/whats-new.

5. The Oxford COVID-19 Government Response Tracker (OxCGRT). https://www.bsg.ox.ac.uk/research/research-proje cts/coronavirus-government-response-tracker.

6. Smythe, T., Kuper, H., Macleod, D., et al. (2017). Birth prevalence of congenital talipes equinovarus in low-and middle-income countries: A systematic review and meta-analysis. Trop Med Int Health, 22(3), 269-285. https://doi.org/10.1111/tmi.12833. 
7. COVID-19 Dashboard Statewise status. https://www.mygov.in/ covid-19. Accessed 10 August 2020.

8. Novel Coronavirus Disease 2019 (COVID-19): Guidelines on rational use of Personal Protective Equipment, Ministry of Health and Family Welfare, Directorate General of Health Services [Emergency Medical Relief]).
Publisher's Note Springer Nature remains neutral with regard to jurisdictional claims in published maps and institutional affiliations. 